JOURNAL OF

SYMPLECTIC GEOMETRY

Volume 2, Number 2, 261-266, 2004

\title{
A NOTE ON MEAN CURVATURE, MASLOV CLASS AND SYMPLECTIC AREA OF LAGRANGIAN IMMERSIONS
}

\author{
Kai Cieliebak and Edward Goldstein
}

In this note we prove a simple relation between the mean curvature form, symplectic area, and the Maslov class of a Lagrangian immersion in a Kähler-Einstein manifold. An immediate consequence is that in Kähler-Einstein manifolds with positive scalar curvature, minimal Lagrangian immersions are monotone.

\section{Introduction}

Let $(M, \omega)$ be a Kähler-Einstein manifold whose Ricci curvature is a multiple of the metric by a real number $\lambda$. In particular, the Kähler form $\omega$ and the first Chern class $c_{1}(M)$ are related by $c_{1}(M)=\frac{\lambda[\omega]}{2 \pi}$ (see Section 3 ). Let $L$ be an immersed Lagrangian submanifold of $M$. Let $H$ be the trace of the second fundamental form of $L$ (the mean curvature vector field of $L$ ). Thus $H$ is a section of the normal bundle to $L$ in $M$ and we have a corresponding 1 -form $\sigma_{L}:=i_{H} \omega$ on $L$, called the mean curvature form of $L$. Consider a smooth map $F: \Sigma \rightarrow M$ from a compact oriented surface $\Sigma$ to $M$ whose (possibly empty) boundary $\partial F:=F(\partial \Sigma)$ is contained in $L$. Let $\mu(F)$ be the Maslov class of $F$ (see Section 2) and $\omega(F):=\int_{\Sigma} F^{*} \omega$ its symplectic area. The goal of this note is to prove the following simple relation between these quantities:

$$
\lambda \omega(F)-\pi \mu(F)=\sigma_{L}(\partial F) .
$$

This relation was given in $[\mathbf{M o r}]$ for $\mathbb{C}^{n}$ and in $[\mathbf{A r s}]$ for Calabi-Yau manifolds. Dazord [Daz] showed that the differential of the mean curvature form is the Ricci form restricted to $L$, so in the Kähler-Einstein case $\sigma_{L}$ is closed (see Section 3). Y.-G. Oh [Oh2] investigated the symplectic area in the case that the mean curvature form is exact.

Lagrangian submanifolds for which $\mu(F)=a \omega(F)$ on all disks $F$, for some $a>0$, are called monotone in the symplectic geometry literature, cf. [Oh1]. 
An immediate consequence of (1) is that in Kähler-Einstein manifolds with positive scalar curvature (i.e. $\lambda>0$ ), minimal (i.e. $\sigma_{L} \equiv 0$ ) Lagrangian immersions are monotone.

In view of the exact sequence in cohomology (with real coefficients)

$$
H^{1}(M) \longrightarrow H^{1}(L) \stackrel{\delta}{\longrightarrow} H^{2}(M, L) \longrightarrow H^{2}(M),
$$

formula (1) can be rephrased as

$$
\lambda[\omega]-\pi \mu=\delta \sigma_{L} \in H^{2}(M, L) .
$$

Note that the class $\lambda[\omega]-\pi \mu$ is equivariant under symplectomorphisms of $M$. It follows that if the map $H^{1}(M) \rightarrow H^{1}(L)$ is trivial, then the cohomology class of the mean curvature form $\sigma_{L}$ is equivariant under symplectomorphisms of $M$. This generalizes Oh's observation [Oh2] that the cohomology class is invariant under Hamiltonian deformations.

Acknowledgement. We thank the anonymous referee for pointing out the generalization (3) of formula (1).

\section{Maslov class}

We first recall a definition of the Maslov index that is suitable for our purposes. Let $V$ be a Hermitian vector space of complex dimension $n$. Let $\Lambda^{(n, 0)} V$ be the (one-dimensional) space of holomorphic $(n, 0)$-forms on $V$ and set

$$
K^{2}(V):=\Lambda^{(n, 0)} V \otimes \Lambda^{(n, 0)} V .
$$

Let $L$ be a Lagrangian subspace of $V$. We can associate to $L$ an element $\kappa(L)$ in $\Lambda^{(n, 0)} V$ of unit length which restricts to a real volume form on $L$. This element is unique up to sign and therefore defines a unique element of unit length

$$
\kappa^{2}(L):=\kappa(L) \otimes \kappa(L) \in K^{2}(V) .
$$

Thus we get a map $\kappa^{2}$ from the Grassmanian $G r_{\text {Lag }}(V)$ of Lagrangian planes to the unit circle in $K^{2}(V)$. This map induces a homomorphism $\kappa_{*}^{2}$ of fundamental groups

$$
\kappa_{*}^{2}: \pi_{1}\left(G r_{\text {Lag }}(V)\right) \rightarrow \mathbb{Z}
$$

To understand the map $\kappa_{*}^{2}$, let $L$ be a Lagrangian subspace and let $v_{1}, \ldots, v_{n}$ be an orthonormal basis for $L$. For $0 \leq t \leq 1$ consider the subspace

$$
L_{t}=\operatorname{span}\left\{v_{1}, \ldots, v_{n-1}, e^{\pi i t} v_{n}\right\} \text {. }
$$

This loop $\left\{L_{t}\right\}$ is the standard generator of $\pi_{1}\left(G r_{\text {Lag }}(V)\right)$. The induced elements in $\Lambda^{(n, 0)} V$ are related by $\kappa\left(L_{t}\right)= \pm e^{-\pi i t} \kappa(L)$, so $\kappa^{2}\left(L_{t}\right)=e^{-2 \pi i t} \kappa^{2}(L)$ and $\kappa_{*}^{2}\left(\left\{L_{t}\right\}\right)=-1$. Thus we see that the homomorphism $\kappa_{*}^{2}$ is related to the Maslov index $\mu$ (as defined, e.g., in $[\mathbf{A u L a}]$ ) by

$$
\kappa_{*}^{2}=-\mu: \pi_{1}\left(G r_{\operatorname{Lag}}(V)\right) \rightarrow \mathbb{Z}
$$


Now let $(M, \omega)$ be a symplectic manifold of dimension $2 n$. Pick an almost complex structure $J$ on $M$ such that $\omega(\cdot, J \cdot)$ defines a Riemannian metric and let $K(M):=\Lambda^{(n, 0)} T^{*} M$ be the canonical bundle of $M$, i.e., the bundle of $(n, 0)$-forms on $M$. Note that $c_{1}(K(M))=-c_{1}(M)$. Let $K^{2}(M):=$ $K(M) \otimes K(M)$ be the square of the canonical bundle.

Let $L$ be an immersed Lagrangian submanifold of $M$. For any point $l \in L$ there is an element of unit length $\kappa(l)$ of $K(M)$ over $l$, unique up to sign, which restricts to a real volume form on the tangent space $T_{l} L$. The squares of these elements give rise to a section of unit length

$$
\kappa_{L}^{2}: L \rightarrow K^{2}(M)
$$

Note that if $L$ is oriented, then $\kappa_{L}^{2}$ is the square of the unit length section $\kappa_{L}: L \rightarrow K(M)$ defined by picking the volume forms $\left.\kappa(l)\right|_{L}$ positive with respect to the orientation.

Now let $F: \Sigma \rightarrow M$ be a smooth map from a compact oriented surface to $M$ with boundary $\partial F=F(\partial \Sigma)$ on $L$. To define the Maslov class $\mu(F)$, assume first that $\Sigma$ is connected and $\partial \Sigma$ is nonempty. Then $H^{2}(\Sigma ; \mathbb{Z})=0$, hence the pullback $F^{*} K(M)$ to $\Sigma$ is a trivial bundle and we can pick a unit length section $\kappa_{F}$ of $K(M)$ over $\Sigma$. Now on the boundary $\partial F$ we also have the section $\kappa_{L}^{2}$ defined above. We can uniquely write

$$
\kappa_{L}^{2}=e^{i \theta} \kappa_{F}^{2}
$$

for a function $e^{i \theta}: \partial \Sigma \rightarrow S^{1}$ to the unit circle. We define the Maslov class $\mu(F)$ as minus its winding number,

$$
\mu(F):=\frac{-1}{2 \pi} \int_{\partial F} d \theta
$$

If $\Sigma$ is closed replace some point of $\Sigma$ by a new boundary circle $\partial \Sigma$ which gets mapped under $F$ to a point $x \in M$. Pick a unit length element $\kappa_{x}$ of $K(M)$ at $x$ and a unit length section $\kappa_{F}$ of $K(M)$ over $\Sigma$ (which is possible since $\Sigma$ now has nonempty boundary). Now write $\kappa_{x}^{2}=e^{i \theta} \kappa_{F}^{2}$ over $\partial \Sigma$ and define $\mu(F):=\frac{-1}{2 \pi} \int_{\partial F} d \theta$ as above. For disconnected $\Sigma$ define $\mu(F)$ as the sum over all connected components.

This definition is independent of the choice of $\kappa_{F}$ and defines a map

$$
\mu: H_{2}(M, L ; \mathbb{Z}) \rightarrow \mathbb{Z}
$$

To see this, first note that any other unit length section $\kappa_{F}^{\prime}$ of $K(M)$ over $F$ is related to $\kappa_{F}$ by a multiple $e^{i \phi}: \Sigma \rightarrow S^{1}$. So on $F(\partial \Sigma)$ we have $\kappa_{L}^{2}=$ $e^{i \theta^{\prime}}\left(\kappa_{F}^{\prime}\right)^{2}$ with $e^{i \theta^{\prime}}=e^{-2 i \phi} e^{i \theta}: \partial \Sigma \rightarrow S^{1}$. By Stokes' theorem, this implies $\int_{\partial F} d \theta^{\prime}=\int_{\partial F} d \theta$. Next suppose that $F$ and $F^{\prime}$ have the same boundary $\partial F=\partial F^{\prime}=: \gamma$ and $\left[F \cup_{\gamma}-F^{\prime}\right]=0 \in H_{2}(M ; \mathbb{Z})$. Then the pullback of $K(M)$ to $\left[F \cup_{\gamma}-F^{\prime}\right]$ is a trivial bundle and there is a unit length section $\kappa$ of $K(M)$ over $\left[F \cup_{\gamma}-F^{\prime}\right]$. If we take the restriction of $\kappa$ to $F$ as $\kappa_{F}$ and the restriction of $\kappa$ to $F^{\prime}$ as $\kappa_{F}^{\prime}$ we get $e^{i \theta}=e^{i \theta^{\prime}}$, and hence $\mu(F)=\mu\left(F^{\prime}\right)$. 
In particular, if $[F]=0 \in H_{2}(M, L ; \mathbb{Z})$ we find an $F^{\prime}: \Sigma^{\prime} \rightarrow L$ with $\partial F=\partial F^{\prime}=\gamma$ and $\left[F \cup_{\gamma}-F^{\prime}\right]=0 \in H_{2}(M ; \mathbb{Z})$, and thus $\mu(F)=\mu\left(F^{\prime}\right)=0$. This shows that $\mu(F)$ depends only on $[F] \in H_{2}(M, L ; \mathbb{Z})$.

In view of the discussion above, our definition of $\mu$ agrees with the usual definition of the Maslov class, cf. [AuLa].

\section{Proof}

Now assume that $(M, \omega)$ is Kähler with complex structure $J$ and Kähler metric $\langle\cdot, \cdot\rangle=\omega(\cdot, J \cdot)$. We denote by $\nabla$ the Levi-Civita connection, as well as the induced connections on $K(M)$ and $K^{2}(M)$. Let us briefly review the geometry of $K(M)$, following the notations in [Bes], pp. 81-82. Any local non-vanishing section $\kappa$ of $K(M)$ over an open subset $U$ of $M$ defines a (complex valued) connection one form $\eta$ on $U$ by $\nabla \kappa=\eta \otimes \kappa$. The curvature of $K(M)$ is defined to be $R_{K}:=-d \eta$; it is a global closed imaginary valued $(1,1)$-form on $M$. By [Bes], Prop. 2.45, the Ricci tensor Ric of $M$ is a symmetric bilinear form of type $(1,1)$; the associated 2 -form $\rho(\cdot, \cdot):=\operatorname{Ric}(J \cdot, \cdot)$ is called the Ricci form of $M$. By [Bes], Prop. 2.96, the Ricci form satisfies

$$
\rho=i R_{K}
$$

If follows (cf. [Bes], Prop. 2.75) that the first Chern class $c_{1}(M)$ is represented by $\frac{\rho}{2 \pi}$. Note that in the Kähler-Einstein case, $\rho=\lambda \omega$.

Now let $L$ be an immersed Lagrangian submanifold of $M$ and let $\kappa_{L}^{2}$ be the canonical section of $K^{2}(M)$ over $L$ as above. The section $\kappa_{L}^{2}$ defines a connection 1-form $\eta_{L}$ for $K^{2}(M)$ over $L$ by the condition $\nabla \kappa_{L}^{2}=\eta_{L} \otimes \kappa_{L}^{2}$. Since $\kappa_{L}^{2}$ has constant length $1, \eta_{L}$ is an imaginary valued 1-form on $L$. Let $\sigma_{L}=i_{H} \omega$ be the mean curvature form of $L$ as in Section 1. The following fact goes back to [Oh2], Prop. 2.2:

$$
\eta_{L}=2 i \sigma_{L}
$$

Here the factor 2 is due to the fact that $\eta_{L}$ is a connection 1-form for $K^{2}(M)$ rather than $K(M)$. In particular, since $d \eta_{L}=-2 R_{K}=2 i \rho$, this formula implies $d \sigma_{L}=\left.\rho\right|_{L}$, so in the Kähler-Einstein case $\sigma_{L}$ is closed.

For the convenience of the reader, we recall the proof of formula (2) from [Gol] (where, however, the formula is stated with the wrong sign). Pick a point $l \in L$ and let $e_{1}, \ldots, e_{n}$ be a local orthonormal frame tangent to $L$. Orient $L$ locally by this frame. Then $\kappa_{L}\left(e_{1}, \ldots, e_{n}\right) \equiv 1$, and hence for every local vector field $v$ tangent to $L$,

$$
0=v\left(\kappa_{L}\left(e_{1}, \ldots, e_{n}\right)\right)=\left(\nabla_{v} \kappa_{L}\right)\left(e_{1}, \ldots, e_{n}\right)+\sum_{j=1}^{n} \kappa_{L}\left(e_{1}, \ldots, \nabla_{v} e_{j}, \ldots, e_{n}\right)
$$


Since the complex structure $J$ is parallel (see [KoNo], Ch. IX Thm. 4.3), the $j$-th term in the last sum equals

$$
i\left\langle\nabla_{v} e_{j}, J e_{j}\right\rangle=i\left\langle\nabla_{e_{j}} v, J e_{j}\right\rangle=-i\left\langle v, J \nabla_{e_{j}} e_{j}\right\rangle=i \omega\left(v, \nabla_{e_{j}} e_{j}\right) .
$$

Summing over $j$ and inserting $H=\sum_{j=1}^{n} \nabla_{e_{j}} e_{j}$, we find

$$
\nabla_{v} \kappa_{L}\left(e_{1}, \ldots, e_{n}\right)=i \sum_{j=1}^{n} \omega\left(\nabla_{e_{j}} e_{j}, v\right)=i \omega(H, v)=i \sigma_{L}(v) .
$$

Now formula (2) follows from $\nabla \kappa_{L}^{2}=\eta_{L} \otimes \kappa_{L}^{2}$ via

$$
\eta_{L}=2 \nabla \kappa_{L}\left(e_{1}, \ldots, e_{n}\right)=2 i \sigma_{L}
$$

Now let $F: \Sigma \rightarrow M$ be a smooth map from a compact oriented surface with boundary on $L$. We will prove the following identity in any Kähler manifold:

$$
\rho(F)-\pi \mu(F)=\sigma_{L}(\partial F) .
$$

Note that in general the form $\sigma_{L}$ need not be closed on $L$. It is closed in the Kähler-Einstein case, in which $\rho=\lambda \omega$ and (3) implies formula (1) in the introduction.

To prove identity (3), assume that every connected component of $\Sigma$ has nonempty boundary (closed components are treated similary, see Section 2). Define the section $\kappa_{F}$ of $K(M)$ over $F$ as in Section 2. Let $\eta_{F}$ be the connection 1-form along $F$ defined by $\nabla \kappa_{F}^{2}=\eta_{F} \otimes \kappa_{F}^{2}$. By the discussion in the beginning of this section, $d \eta_{F}=2 i F^{*} \rho$. Stokes' theorem implies

$$
2 \rho(F)=\int_{\partial F}-i \eta_{F}
$$

Recall from Section 2 that along $\partial F$ we have $\kappa_{L}^{2}=e^{i \theta} \kappa_{F}^{2}$ for a function $e^{i \theta}: \partial \Sigma \rightarrow S^{1}$, and the Maslov class is given by

$$
\mu(F)=\frac{-1}{2 \pi} \int_{\partial F} d \theta
$$

The connection 1-forms $\eta_{F}$ and $\eta_{L}$ are related by

$$
\eta_{L}=\eta_{F}+i d \theta
$$

on $\partial F$. Combining the equations above and formula (2), we find

$$
\sigma_{L}(\partial F)=\int_{\partial F} \frac{-i \eta_{L}}{2}=\int_{\partial F} \frac{-i \eta_{F}}{2}+\int_{\partial F} \frac{d \theta}{2}=\rho(F)-\pi \mu(F) .
$$




\section{References}

[Ars] A. Arsie, Maslov class and minimality in Calabi-Yau manifolds, J. Geom. Phys. 35, no. 2-3, 145-156 (2000).

[AuLa] M. Audin and J. Lafontaine (editors), Holomorphic Curves in Symplectic Geometry, Progress in Math. 117, Birkhäuser, Basel (1994).

[Bes] A. Besse, Einstein manifolds, Ergebnisse der Mathematik und ihrer Grenzgebiete, 3. Folge Band 10.

[Daz] P. Dazord, Sur la géometrie des sous-fibrés et des feuilletages lagrangiens, Ann. Sci. Ec. Norm. Super. IV, Sr. 13, 465-480 (1981).

[Gol] E. Goldstein, A construction of new families of minimal Lagrangian submanifolds via torus actions, J. Diff. Geom. 58, 233-261 (2001).

[KoNo] S. Kobayashi and K. Nomizu, Foundations of Differential Geometry, Volume II, John Wiley (1969).

[Mor] J.-M. Morvan, Classe de Maslov d'une immersion lagrangienne et minimalité, C.R. Acad. Sci. 292, 633-636 (1981).

[Oh1] Y.-G. Oh, Floer cohomology of Lagrangian intersections and pseudo-holomorphic disks I, Comm. Pure Appl. Math. 46, no. 7, 949-993 (1993).

[Oh2] Y.-G. Oh, Mean curvature vector and symplectic topology of Lagrangian submanifolds in Einstein-Kähler manifolds, Math. Z. 216, 471-482 (1994).

Mathematisches Institut Zimmer 313, Ludwig-Maximilians-Universitaet, ThereSienstr. 39, 80333 Muenchen, Germany

E-mail address: kai@mathematik.uni-muenchen.de

Department of Mathematics, Stanford University, 450 Sierra Mall, Bldg. 380, STANFORD, CA 94305-2125

E-mail address: egold@math.stanford.edu 